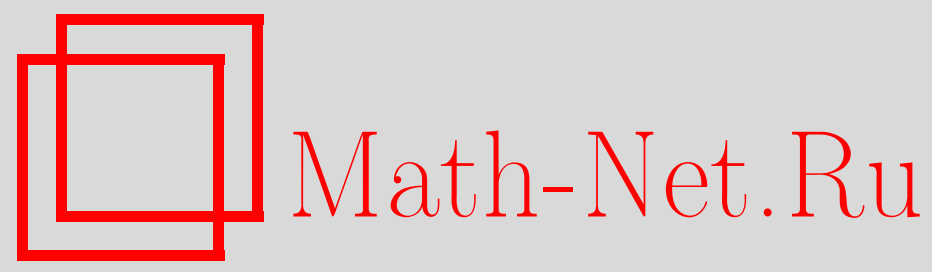

А. Дубицкас, Многочлены с большой кратностью в единице и проблема Терри, Матем. заметки, 1999, том 65, выпуск $6,810-815$

DOI: https://doi.org/10.4213/mzm1116

Использование Общероссийского математического портала Math-Net.Ru подразумевает, что вы прочитали и согласны с пользовательским соглашением http://www . mathnet.ru/rus/agreement

Параметры загрузки:

IP: 107.22 .136 .117

26 апреля 2023 г., 14:11:19

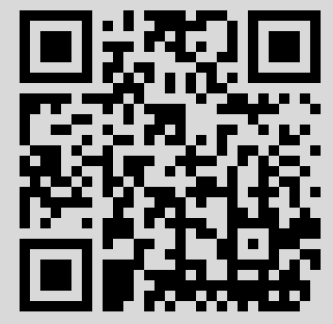




\section{МНОГОЧЛЕНЫ С БОЛЬШОЙ КРАТНОСТЬЮ В ЕДИНИЦЕ И ПРОБЛЕМА ТЕРРИ}

\section{А. Дубицкас}

Устанавлено существование многочлена с цельми коэффициентами, который имеет относительно небольшую длину и достаточно большую кратность в единице. Доказательство соответствующего утверждения основано на оценке числа решений системы Терри.

Библиографоя: 14 названий.

Пусть $P(z)$ - ненулевой многочлен с цельми коэффициентами степени $\operatorname{deg} P(z) \leqslant N-$ 1. Обозначим кратность многочлена в единище через

$$
r(P)=\operatorname{ord}_{z=1} P(z)
$$

Пусть также $H(P)$ и $L(P)$ являются соответственно высотой и длиной многочлена $P(z)$. Из классической леммы Зигеля следует, что существуют многочлены с не очень большой высотой и достаточно большой кратностью в единице. Так, Блох и Пойя [1] показали, что существует многочлен с коэффищиентами $\{0,1,-1\}$ такой, что

$$
r(P) \geqslant\left[\sqrt{2 \log 2 \frac{N-1}{\log N}}\right]-1 .
$$

Миньотт [2] получил соответствующую оценку снизу для многочлена с произвольной, но не очень большой высотой. Эти результаты, обычно назьваемые вариантами леммы Зигеля, основаны на применении принципа Дирихле. Иногда для приложений требуются как можно более точные варианты леммы Зигеля. Так, например, Бомбьери [3] использовал подобные результаты для оценок эффективных приближений алгебраических чисел. В работе [4] Бомбьери и Ваалер получили общий вариант леммы Зигеля. Вместо принципа Дирихле они воспользовались рядом результатов из геометрии чисел. Ряд специальных важных случаев рассматривался ими в работе [5]. В частности, они показали, что существует многочлен с высотой $\leqslant H$ такой, что

$$
\frac{\log H}{N} \leqslant \frac{1}{2}\left(\frac{r(P)}{N}\right)^{2} \log \left(\frac{N}{r(P)}\right)(1+\varepsilon),
$$

где $\varepsilon>0$, а $N / r(P)$ достаточно большое.

Работа выполнена при частичной поддержке Литовского фонда студий и науки. 
Легко видеть, что из (2) следует существование многочлена с коэффициентами $\{0$, $1,-1\}$ такого, что

$$
r(P) \geqslant(1-\varepsilon) \sqrt{4 \log 2 \frac{N}{\log N}},
$$

где $\varepsilon>0$, а $N$ достаточно большое. Ясно, что неравенство (3) усиливает неравенство (1).

Недавно Аморозо [6], используя идеи Добровольского, разработанные при исследовании гипотезы Лемера [7], показал, что константа $1 / 2$ в (2) может быть заменена на $1 / 4$ при вьполнении некоторых дополнительных условий. Однако, для этого требуется, чтобы высота была достаточно большая, т.е. это не дает усиления неравенства (3). Наконец, автор [8], [9] рассматривал явно построенный многочлен с большой кратностью в единище, для которого вьполняется неравенство типа (2).

Хорошо известно, что кратность многочлена в единице не может быть очень большой, если его высота или длина небольшие. По теореме Шура [10] количество вещественных корней многочлена $P$ не превосходит $2 \sqrt{N \log L(P)}$. Следовательно, справедливо неравенство

$$
r(P) \leqslant 2 \sqrt{N \log L(P)} .
$$

В том случае, когда $H(P)$, а вместе с тем и $L(P)$, достаточно большое, неравенство (4) также было доказано Бомбьери и Ваалером [5]. Аморозо [6] доказал, что константа 2 в (4) может быть заменена на 1.21 , когда $N$ и $\log H(P) / \log N$ достаточно большие. Заметим, что в случае $L(P) \leqslant N$ из (4) следует, что

$$
r(P) \leqslant 2 \sqrt{N \log N}
$$

Таким образом, если неравенство (3) может быть уточнено, то только за счет константы и $\log N$.

В настоящей работе мы покажем, что существуют многочлены с небольшой по сравнению со степенью длиной, имеющие достаточно большую кратность в единице. Справедлива следующая

TЕоремА. Для любого $\varepsilon>0$ существует $\delta=\delta(\varepsilon)>0$ такое, что если $1 /(\delta N) \leqslant$ $a \leqslant 2^{N-1}$, то существует многочлен с иелыми коэффициентами $P$, удовлетворяюший условиям $\operatorname{deg} P \leqslant N-1, L(P) \leqslant a N$,

$$
r(P)>(\sqrt{2}-\varepsilon) \sqrt{\frac{f(a) N}{\log N}},
$$

где $f(a)=(1+a / 2) \log (1+a / 2)-(a / 2) \log (a / 2)$. Более того, если $N^{-\delta} \leqslant a \leqslant 2^{N-1}$ и $N$ достаточно большое, то

$$
r(P)>(2-\varepsilon) \sqrt{\frac{f(a) N}{\log N}} .
$$

Отметим, что если $a$ - целое положительное число, то при условиях теоремы из (2) следует неравенство

$$
r(P) \geqslant(2-\varepsilon) \sqrt{\frac{N \log (1+a)}{\log N}} .
$$


Если же $a<1$, то при условиях теоремы из (3) следует неравенство

$$
r(P) \geqslant(1-\varepsilon) \sqrt{4 \log 2 \frac{a N}{\log (a N)}}
$$

Нижние оценки для $r(P)$ в теореме сильнее, чем (7) и (8). Например, при $a=1$ неравенство (6) сильнее, чем (7):

$$
f(1)=\frac{3}{2} \log 3-\log 2>\log 2,
$$

а при $a=1 / \sqrt{N}$ неравенство (5) лучше, чем (8), на логарифмический множитель $\sqrt{\log N}$. Если $a \geqslant 2^{N-1} / N$, то многочлен $(1-z)^{N-1}$ имеет максимально возможную кратность $r(P)=N-1$.

Первоначальный вариант доказательства теоремы с функцией несколько меньшей, чем $f(a)$ состоял в применении оценок, получаемых в проблеме Терри (или Пруэ-Терри-Эскотта). Рассмотрим систему из $n$ уравнений

$$
x_{1}^{j}+x_{2}^{j}+\cdots+x_{k}^{j}-y_{1}^{j}-y_{2}^{j}-\cdots-y_{k}^{j}=0, \quad j=1,2, \ldots, n,
$$

где неизвестные $x_{1}, x_{2}, \ldots, x_{k}, y_{1}, y_{2}, \ldots, y_{k}$ принимают значения целых чисел от 1 до $N$. Различные вопросы о числе решений этой системы обычно называют проблемой Терри (см., например, [11]-[13] или [14, гл. III, лемма 5], где приводится оценка снизу для числа решений этой системы). Решение системы Терри $\left(x_{1}, x_{2}, \ldots, x_{k}, y_{1}, y_{2}, \ldots, y_{k}\right)$ назовем тривиальным, если числа $x_{1}, x_{2}, \ldots, x_{k}$ получаются перестановкой чисел $y_{1}, y_{2}, \ldots, y_{k}$.

Рассмотрим многочлен

$$
F(z)=\sum_{j=1}^{k} z^{x_{j}}-\sum_{j=1}^{k} z^{y_{j}}
$$

где $\left(x_{1}, x_{2}, \ldots, x_{k}, y_{1}, y_{2}, \ldots, y_{k}\right)$ - нетривиальное решение системы Терри. Легко видеть, что

$$
F(1)=F^{\prime}(1)=\cdots=F^{(n)}(1)=0 .
$$

Положим $P(z)=F(z) / z$. Ясно, что $P(z)$ - ненулевой многочлен с целыми коэффициентами степени $\leqslant N-1$. Кроме того, $L(P)=L(F) \leqslant 2 k$ и $r(P)=r(F) \geqslant n+1$. Для доказательства теоремы остается проверить, что при $k=[a N / 2]$ и достаточно больших $r$ (соответствующих (5), (6)) система Терри (9) с $n=r-1$ имеет нетривиальное решение.

Автор благодарит рецензента, которьй предложил дать непосредственное доказательство этого и не пользоваться известными нижними оценками для числа всех решений системы Терри, которые хороши только тогда, когда $N$ велико по сравнению с $n$ и $k$.

ДоКАЗАТЕЛЬСТВо. Ясно, что при $n=r-1$ система (9) эквивалентна следующей:

$$
\left(\begin{array}{c}
x_{1} \\
j
\end{array}\right)+\left(\begin{array}{c}
x_{2} \\
j
\end{array}\right)+\cdots+\left(\begin{array}{c}
x_{k} \\
j
\end{array}\right)-\left(\begin{array}{c}
y_{1} \\
j
\end{array}\right)-\left(\begin{array}{c}
y_{2} \\
j
\end{array}\right)-\cdots-\left(\begin{array}{c}
y_{k} \\
j
\end{array}\right)=0, \quad j=1,2, \ldots, r-1 .
$$


Рассмотрим все $(r-1)$-мерные векторы

$$
\left(\sum_{i=1}^{k} x_{i}, \sum_{i=1}^{k}\left(\begin{array}{c}
x_{i} \\
2
\end{array}\right), \ldots, \sum_{i=1}^{k}\left(\begin{array}{c}
x_{i} \\
r-1
\end{array}\right)\right)
$$

где $1 \leqslant x_{i} \leqslant N$. Ясно, что $j$-я компонента этих векторов принимает неотрицательные целочисленные значения, не превосходяшие $k\left(\begin{array}{c}N \\ j\end{array}\right)$. Кроме того, первая компонента принимает лиш положительные значения, а при $r \leqslant N / 2 j$-я компонента $(1 \leqslant j \leqslant r-1)$ не равна, скажем, $k\left(\begin{array}{c}N \\ j\end{array}\right)-1$. Поэтому общее количество различных векторов не превосходит

$$
\prod_{j=1}^{r-1} k\left(\begin{array}{c}
N \\
j
\end{array}\right) \leqslant k^{r-1} N^{r(r-1) / 2} \prod_{j=1}^{r-1}(j !)^{-1} .
$$

Используя неравенство $j !>(j / e)^{j}$ и индукцию по $r$, имеем

$$
\prod_{j=1}^{r-1} j !>\frac{r^{2}(\log r-2)}{2}
$$

Следовательно, количество различных векторов не превосходит

$$
k^{r-1}\left(\frac{8 N}{r}\right)^{r^{2} / 2}<(a N)^{r}\left(\frac{8 N}{r}\right)^{r^{2} / 2}
$$

Если это меньше количества неупорядоченньх наборов $\left\{x_{1}, x_{2}, \ldots, x_{k}\right\}$, т.е. меньше $\left(\begin{array}{c}N+k-1 \\ k\end{array}\right)$, то хотя бы два вектора совпадают при различных наборах, и система Терри имеет нетривиальное решение.

Для доказательства теоремы остается проверить неравенство

$$
(a N)^{r}\left(\frac{8 N}{r}\right)^{r^{2} / 2}<\left(\begin{array}{c}
N+[a N / 2]-1 \\
N-1
\end{array}\right)
$$

где

$$
r=\left(\theta-\frac{\varepsilon}{2}\right) \sqrt{\frac{f(a) N}{\log N}}
$$

а $\theta$ равно соответственно $\sqrt{2}$ или 2 . В обоих случаях теоремы числа $N$ и $a N$ достаточно большие, поэтому с помощью формулы Стирлинга

$$
e^{-1 /(12 j)}<\frac{j !}{\sqrt{2 \pi j}(j / e)^{j}}<e^{1 /(12 j)}
$$

легко получить оценку снизу для биномиального коэффициента

$$
\begin{aligned}
\left(\begin{array}{c}
N+[a N / 2]-1 \\
N-1
\end{array}\right) & =\frac{N}{N+[a N / 2]} \frac{(N+[a N / 2]) !}{N ![a N / 2] !} \\
& >\frac{1}{(N+a N / 2)^{3}}\left(\frac{(1+a / 2)^{1+a / 2}}{(a / 2)^{a / 2}}\right)^{N}=\frac{1}{(N+a N / 2)^{3}} e^{f(a) N} .
\end{aligned}
$$


Прологарифмируем неравенство (10) и проверим, что

$$
(r+3) \log (N+a N)+\frac{r^{2}}{2} \log \left(\frac{8 N}{r}\right)<f(a) N .
$$

Возьмем достаточно малое $\delta>0$, и пусть $N$ достаточно большое. В обоих случаях $\left(1 /(\delta N) \leqslant a \leqslant 2^{N-1}\right.$ и $\left.N^{-\delta} \leqslant a \leqslant 2^{N-1}\right)$ имеем

$$
(r+3) \log (N+a N)=\left(\left(\theta-\frac{\varepsilon}{2}\right) \sqrt{\frac{f(a) N}{\log N}}+3\right) \log (N+a N)<\frac{\varepsilon}{8} f(a) N .
$$

Ясно также, что число $r$ достаточно большое, так как

$$
f(a)>\frac{a}{2} \log \left(1+\frac{2}{a}\right)>\frac{\log (\delta N)}{2 \delta N} .
$$

Поэтому если $1 /(\delta N) \leqslant a$ и $\varepsilon$ достаточно мало, то

$$
\frac{r^{2}}{2} \log \left(\frac{8 N}{r}\right)<\frac{r^{2}}{2} \log N=\frac{1}{2}(\sqrt{2}-\varepsilon)^{2} f(a) N<(1-\varepsilon) f(a) N .
$$

Следовательно, неравенство (11) вьполняется, что доказьвает (5).

Если $a \geqslant N^{-\delta}$ и $N$ достаточно большое, то

$$
f(a)>\frac{a}{2} \log \left(1+\frac{2}{a}\right)>\frac{\delta}{2} \frac{\log N}{N^{\delta}} .
$$

Поэтому $r$ можно оценить снизу

$$
r=\left(2-\frac{\varepsilon}{2}\right) \sqrt{\frac{f(a) N}{\log N}}>N^{1 / 2-\delta} .
$$

Следовательно, $8 N / r<N^{1 / 2-2 \delta}$ и

$$
\frac{r^{2}}{2} \log \left(\frac{8 N}{r}\right)<\left(\frac{1}{4}-\delta\right) r^{2} \log N<\left(\frac{1}{4}-\delta\right)\left(4-\frac{\varepsilon}{2}\right) f(a) N<\left(1-\frac{\varepsilon}{8}\right) f(a) N .
$$

Теорема доказана, так как неравенство (11) по-прежнему вьполняется.

Подобным образом можно доказать и неравенство (3). Пусть все $x_{1}, x_{2}, \ldots, x_{k}$ различны. Число таких наборов равно $\left(\begin{array}{l}N \\ k\end{array}\right)$, а вместо (10) теперь имеем неравенство

$$
k^{r}\left(\frac{8 N}{r}\right)^{r^{2} / 2}<\left(\begin{array}{c}
N \\
k
\end{array}\right) \text {. }
$$

Для любого $\varepsilon, 0<\varepsilon<1 / 2$, и достаточно большого $N$ это неравенство выполняется, если взять $k=[N / 2]$ и

$$
r=\left(1-\frac{\varepsilon}{2}\right) \sqrt{4 \log 2 \frac{N}{\log N}} .
$$

Несколько более сложными комбинаторными рассуждениями можно сосчитать количество наборов $\left\{x_{1}, \ldots, x_{k}\right\}$, где среди $x_{1}, \ldots, x_{k}$ не более, чем $H$ одинаковых чисел. Выбирая $k$ оптимальным образом, получим неравенство Бомбьери и Ваалера (2). 


\section{СПИСОК ЦИТИРОВАННОЙ ЛИТЕРАТУРЫ}

[1] Bloch A., Pólya G. On the roots of certain algebraic equations // Proc. London Math. Soc. 1932. V. 33. P. 102-114.

[2] Mignotte M. Sur la répartition des racines des polynômes // J. Théor. Anal. Elém. Nombres. Caen, 29-30 septembre 1980. P. 24-34.

[3] Bombieri E. On the Thue-Siegel theorem // Acta Math. 1982. V. 148. P. 255-296.

[4] Bombieri E., Vaaler J. On Siegel's lemma // Invent. Math. 1983. V. 73. P. 11-32.

[5] Bombieri E., Vaaler J. Polynomials with low height and prescribed vanishing // Analytic Number Theory and Diophantine Problems / ed. A. C. Adolphson, J. B. Conrey, A. Ghosh, R. I. Yager. Progr. Math. V. 70. Boston: Birkhäuser, 1987. P. 53-73.

[6] Amoroso F. Polynomials with prescribed vanishing at roots of unity // Boll. Un. Mat. Ital. B (7).. 1995. V. 9. № 7. P. 1021-1042.

[7] Dobrowolski E. On a question of Lehmer and the number of irreducible factors of a polynomial // Acta Arith. 1979. V. 34. P. 391-401.

[8] Dubickas A. On algebraic numbers of small measure // Lithuanian Math. J. 1995. V. 35. P. 333-342.

[9] Dubickas A. On a polynomial with large number of irreducible factors (to appear).

[10] Schur I. Untersuchungen über algebraische Gleichungen. I. Bemerkungen zu einem Satz von E. Schmidt // S. -B. Preuss. Akad. Wiss. 1933. H. 7/10. P. 403-428.

[11] Виноградов И. М. Метод тригонометрических сумм в теории чисел. М.: Наука, 1980.

[12] Хуа Ло-ген. Метод тригонометрических сумм и его применения в теории чисел. М.: Наука, 1964.

[13] Vooley T. Some remarks on Vinogradov's mean value theorem and Tarry's problem // Monatsh. Math. 1996. V. 122. P. 265-273.

[14] Архипов Г.И., Карацуба А. А., Чубариков В.Н. Теория кратных тригонометрических сумм. М.: Наука, 1987. 